\title{
Der pädiatrische Notfall Überblick über die wichtigsten Erkrankungen
}

Johannes Pöschl

Nicht nur Berufsanfänger empfinden Kindernotfälle als schwierig. Selbst erfahrene Notärzte werden mitunter unsicher - vor allem, wenn sie kleinere Kinder behandeln müssen. Dafür gibt es zwei Gründe: Pädiatrische Notfälle sind selten, und meist handelt es sich um gänzlich andere Krankheitsbilder als bei Erwachsenen. Viel Gelegenheit zum Üben werden Sie daher vermutlich nicht erhalten. Umso besser, wenn Sie wissen, was im Ernstfall auf Sie zukommt. Im Folgenden stellen wir Ihnen die Kindernotfälle vor, mit denen Sie am ehesten konfrontiert werden und deren Therapie Sie auch als Nicht-Pädiater beherrschen sollten.

Tab. 1 Mod. nach [1]. WBC: weiße Blutkörperchen.

Prinzipielle Behandlungsstrategie Auch wenn Sie bei einem Kindernotfall unsicher sind: In der Notfallmedizin geht es letztlich darum, dass der Patient genug Luft bekommt, einen funktionierenden Kreislauf hat, bei Bewusstsein und schmerzfrei ist. Daraus lassen sich die häufigsten Leitsymptome den unterschiedlichen Diagnosen zuordnen. Das ist bei Kindern nicht anders.

Zuerst müssen Sie also die Schwere der Krankheit beurteilen. Als Kriterien dienen:

- das Ausmaß der Dyspnoe,

- das Aussehen der Haut (blass, zyanotische oder extrem gestörte Mikrozirkulation), aber auch

$\checkmark$ die Bewusstseinslage.

\begin{tabular}{|c|c|c|c|c|c|c|}
\hline \multicolumn{7}{|c|}{ Pathologische Grenzwerte bestimmter Vital- und Laborparameter bei Kindern } \\
\hline Altersgruppe & $\begin{array}{l}\text { Hypotonie } \\
\text { (RR systol.) } \\
\text { [mmHg] }\end{array}$ & $\begin{array}{l}\text { Brady- } \\
\text { kardie } \\
{\left[\mathrm{min}^{-1}\right]}\end{array}$ & $\begin{array}{l}\text { Tachy- } \\
\text { kardie } \\
{\left[\mathrm{min}^{-1}\right]}\end{array}$ & $\begin{array}{l}\text { Tachy- } \\
\text { pnoe } \\
{\left[\mathrm{min}^{-1}\right]}\end{array}$ & $\begin{array}{l}\text { Leuko- } \\
\text { penie } \\
{[\mathrm{WBC} / \mathrm{nl}]}\end{array}$ & $\begin{array}{l}\text { Leuko- } \\
\text { zytose } \\
\text { [WBC/nl] }\end{array}$ \\
\hline $\begin{array}{l}\text { Neugeborene } \\
0 \text { bis }<8 \text { Tage } \\
8 \text { bis }<29 \text { Tage }\end{array}$ & $\begin{array}{l}<65 \\
<75\end{array}$ & $\begin{array}{l}<100 \\
<100\end{array}$ & $\begin{array}{l}>180 \\
>180\end{array}$ & $\begin{array}{l}>50 \\
>40\end{array}$ & $\begin{array}{l}<9,0 \\
<5,0\end{array}$ & $\begin{array}{l}>34,0 \\
>19,5\end{array}$ \\
\hline $\begin{array}{l}\text { Säuglinge } \\
29 \text { Tage bis < } 1 \text { Jahr }\end{array}$ & $<100$ & $<90$ & $>180$ & $>34$ & $<5,0$ & $>17,5$ \\
\hline $\begin{array}{l}\text { Kleinkinder } \\
1 \text { bis }<6 \text { Jahre }\end{array}$ & $<95$ & N.D. & $>140$ & $>22$ & $<6,0$ & $>15,5$ \\
\hline $\begin{array}{l}\text { Schulkinder } \\
6 \text { bis }<12 \text { Jahre }\end{array}$ & $<105$ & N.D. & $>130$ & $>18$ & $<4,5$ & $>13,5$ \\
\hline $\begin{array}{l}\text { Jugendliche } \\
12 \text { bis < } 18 \text { Jahre }\end{array}$ & $<117$ & N.D. & $>110$ & $>14$ & $<4,5$ & $>11,0$ \\
\hline
\end{tabular}

Kindliche Besonderheiten Geht es an die weitere Untersuchung und die Therapie, müssen Sie die physiologischen, anatomischen und psychischen Besonderheiten von Kindern unterschiedlicher Altersklassen jedoch bedenken.

- Nicht nur die Vitalparameter ändern sich mit dem Alter ( Tab. 1), auch

- Medikamente sind oft altersabhängig und immer gewichtsabhängig zu dosieren. Hierbei hilft die Formel zur Abschätzung des Körpergewichts $(K G)$ : $K G=2 \times$ Lebensalter [in Jahren] +8

- Anatomische Kenntnisse sind bei speziellen Maßnahmen, wie z.B. der Intubation, wichtig: So ist bei Kindern der Ringknorpel die engste Stelle, und der Kehlkopf liegt höher.

- Beachten Sie außerdem, dass die Eltern der Kinder meist sehr besorgt sind und v.a. kleine Kinder den Arzt als Bedrohung empfinden können. Gerade bei Schmerzen oder Spritzen reagieren sie weniger rational als Erwachsene.

Anders als bei Erwachsenennotfällen stehen im Kindesalter respiratorische Probleme, Dehydratation und Krämpfe im Vordergrund. 


\section{Respiratorische Notfälle}

Charakteristik Respiratorische Notfälle sind durch die Symptome Stridor und Dyspnoe gekennzeichnet. Die Art des Stridors kann dabei bereits wichtige Hinweise ergeben. Bei einer Obstruktion im Bereich der extrathorakalen Trachea entsteht ein inspiratorischer Stridor - bei einer intrathorakalen Einengung ein exspiratorischer oder kombinierter Stridor.

\section{Inspiratorische Probleme}

Pseudokrupp ist am wahrscheinlichsten Der häufigste inspiratorische Stridor ist der Pseudokrupp (stenosierende Laryngotracheitis). Er ist gekennzeichnet durch eine subglottische entzündliche Schwellung der Luftwegeschleimhaut - ohne dass ein Atemwegsinfekt vorliegen muss. Die betroffenen Kinder weisen meist eine charakteristische Symptom-Trias auf, bestehend aus

- bellendem Husten,

- Heiserkeit und

- inspiratorischem Stridor.

Ausprägung der Symptome variiert Der Pseudokrupp tritt überwiegend bei Kindern zwischen dem 6. Lebensmonat und 3 Jahren auf. Der Schweregrad der Erkrankung ist durchaus variabel und reicht vom typischen Husten bis hin zu Herzrhythmusstörungen und Somnolenz.

- Schweregrad I: bellender Husten, Heiserkeit, leiser Stridor bei Erregung

- Schweregrad II: Ruhestridor, beginnende Dyspnoe, leichte juguläre Einziehungen

- Schweregrad III: Dyspnoe in Ruhe, ausgeprägte interkostale Einziehungen, Blässe, Tachykardie $>160 /$ min

- Schweregrad IV: hochgradige Dyspnoe mit zunehmender Ateminsuffizienz und Zyanose, Bradykardie und Somnolenz

Adrenalin hilft Therapeutisches Mittel der Wahl ist

- in leichten Fällen inhalatives Adrenalin oder die systemische Gabe von Steroiden (z. B. Prednison $100 \mathrm{mg}$ supp.).

- In schweren Fällen können bis zu $5 \mathrm{ml}$ einer 1:1000-Adrenalin-Lösung inhaliert und zusätzlich 2 Hübe Epinephrin-Pumpspray $(=0,4 \mathrm{mg}$ Adrenalin) verabreicht werden.

Der Nutzen von warmer oder kalter feuchter Luft ist in der Literatur umstritten.

Kinder, die Adrenalin und Steroide erhalten, sollten stationär überwacht werden, da beim Nachlassen der Wirkung Rezidive auftreten können.

Selten: Epiglottitis Durch die Einführung der HIB-Impfung (HIB: Haemophilus influenzae Typ
B) ist das schwere Krankheitsbild der bakteriellen Laryngotracheobronchitis (Epiglottitis) äußerst selten geworden. Denken Sie trotzdem daran bei

- schwerkrankem Kind mit

- hohem Fieber,

- ausgeprägter Schluckstörung,

- kloßiger Sprache und

- Speichelfluss.

Cave Bei Verdacht auf Epiglottitis darf eine Racheninspektion nur in Intubationsbereitschaft auf der Intensivstation erfolgen. Es besteht die Gefahr eines reflektorischen Atem- oder Herzstillstands.

\section{Asthma bronchiale und obstruktive Bronchitis \\ $\nabla$}

Daran erkennen Sie die Obstruktion Eine hochgradige Obstruktion der kleinen Atemwege mit exspiratorischem Stridor ist meist Ausdruck einer Bronchiolitis oder eines Asthma bronchiale. Die klinischen Leitsymptome sind

- Giemen und ein

- verlängertes Exspirium.

Zeichen der schweren bronchialen Obstruktion sind

- kaum hörbares Atemgeräusch,

- Zyanose,

- $\mathrm{CO}_{2}$-Retention sowie

- Verwirrtheit und Eintrübung.

Das Bild der Hypoxie und Hyperkapnie tritt im Verlauf erst spät auf.

Behandlung Therapeutisch sind eine Sauerstoffgabe und die Inhalation von $\beta 2$-Mimetika, Epinephrin, Steroiden und ggf. Theophyllin essenziell. Lässt sich eine Intubation nicht umgehen, sollte Ketamin den Vorzug erhalten - Barbiturate oder Opiate sind dagegen besser zu vermeiden.

Im Akutfall sollten Sie

- den Oberkörper des Kindes aufrecht lagern,

- Kind und Eltern beruhigen,

- Sauerstoff über eine Nasensonde (2-41/min) zuführen,

- eine Inhalationstherapie einleiten und

- Prednison $100 \mathrm{mg}$ rektal bzw. Prednisolon 2-3 mg/kg KG p.o./i.v. zur Entzündungshemmung verabreichen.

Inhalationstherapie Ziel der Inhalation ist das Abschwellen der Schleimhäute. Geeignet sind:

- 10 Trpf. Salbutamol 0,5\% in $2 \mathrm{ml} \mathrm{NaCl} \mathrm{0,9 \%} \mathrm{oder}$

- alternativ 2-4 Hübe Salbutamol-Dosieraerosol, ggf. mit Wiederholung alle 20-30 min (max. alle $10 \mathrm{~min}$ ).

- Versuchsweise ist die Gabe von 10-20Trpf. Ipratropiumbromid p.i. möglich,

- bei Säuglingen Adrenalin 1:1000 in 2-3 ml $\mathrm{NaCl} 0,9 \%$ p.i. 
Was tun, wenn die Medikation nicht wirkt? Wenn die inhalative Behandlung nicht anschlägt, ist eine stationäre Aufnahme mit ggf. intensivmedizinischer Überwachung indiziert. Die weitere Therapie beinhaltet u.a.:

- Theophyllin i.v. (initial: $5 \mathrm{mg} / \mathrm{kg}$ KG i.v., dann $20 \mathrm{mg} / \mathrm{kg} / \mathrm{d}$ als Dauerinfusion, Spiegelkontrolle; bei bestehender Dauertherapie ohne initialen Bolus)

- Salbutamol i.v. $(0,1(-1) \mu \mathrm{g} / \mathrm{kg} / \mathrm{min}$ als Dauerinfusion)

- bei Erschöpfung die maschinelle Beatmung

\section{Differenzialdiagnose: Aspiration}

Symptome Eine wichtige Differenzialdiagnose sowohl des inspiratorischen als auch des expiratorischen Stridors ist die Fremdkörperaspiration. Ein konkretes Aspirationsereignis wird jedoch nur selten beobachtet. Auffällig sind

- Husten und/oder Atemnot

- ohne Fieber und Asthmaanamnese.

So entfernen Sie den Fremdkörper Bei kritischer Verlegung der Atemwege sind zunächst

- thorakale Kompressionen im 1. Lebensjahr und

- thorakale oder abdominelle Kompressionen beim älteren Kind indiziert.

Gelingt es dadurch nicht, einen wirksamen Hustenstoß zu induzieren, kann man

- den pharyngealen Fremdkörper durch eine frühzeitige direkte Laryngoskopie mit einer Magillzange entfernen.
Sitzt der Fremdkörper nicht sichtbar subglottisch, müssen Sie intubieren, um den Fremdkörper in einen Hauptbronchus vorschieben und bronchoskopisch extrahieren zu können. Eine partielle Ventilation der Lunge reicht meist zur Oxygenierung aus.

\section{Unausgeglichener Wasserhaushalt}

\section{Exsikkose}

$\nabla$

Großer Flüssigkeitsverlust möglich Im Rahmen einer akuten Gastroenteritis kann es zur Dehydratation kommen. Diese kann unabhängig vom Erreger hypo-, iso- oder hyperton sein.

- Die Dehydratation kann das 1- bis 3-Fache des zirkulierenden Blutvolumens betragen (80$250 \mathrm{ml} / \mathrm{kg} \mathrm{KG}$ ).

Der Gewichtsverlust lässt sich über das aktuelle Vorgewicht direkt ermitteln oder anhand 0 Tab. 2 abschätzen.

Orale Rehydrierung Bei leichter bis mäßiger Dehydratation und bei allen Kindern jenseits der Neugeborenen-Periode gilt:

- Wenn immer möglich, rehydrieren Sie den Patienten oral.

- Ziel ist es, den Flüssigkeitsverlust innerhalb von $8 \mathrm{~h}$ über eine Magensonde auszugleichen.

Dazu werden nach Vorgabe der WHO hypotone Lösungen mit einem Natriumgehalt von 40$60 \mathrm{mmol} \mathrm{Na} / \mathrm{l}$ verwendet.

Parenterale Rehydrierung Zur intravenösen Rehydrierung nutzt man bei allen Kindern jenseits der Neugeborenen-Periode primär $\mathrm{NaCl}$ 0,9\%.

Tab. 2 Nach [2].

\section{Schweregrade der Dehydratation}

\begin{tabular}{|c|c|c|c|}
\hline Parameter & $\begin{array}{l}\text { keine oder minimale } \\
\text { Dehydratation }\end{array}$ & $\begin{array}{l}\text { leichte bis mittelschwere } \\
\text { Dehydratation }\end{array}$ & schwere Dehydratation \\
\hline Gewichtsverlust & $<5 \%$ & $5-10 \%$ & $\geq 10 \%$ \\
\hline $\begin{array}{l}\text { Allgemeinzustand, } \\
\text { Bewusstsein }\end{array}$ & gut, wach & unruhig, irritabel oder müde & $\begin{array}{l}\text { apathisch, lethargisch, } \\
\text { bewusstlos }\end{array}$ \\
\hline Durst & normal & durstig, gierig zu trinken & $\begin{array}{l}\text { trinkt schlecht oder kann nicht } \\
\text { mehr trinken }\end{array}$ \\
\hline Schleimhäute (Mund, Zunge) & feucht & trocken & ausgetrocknet \\
\hline Rekapillarisierung & normal $(<2 \mathrm{~s})$ & verlängert (>2s) & verlängert (> 2s) \\
\hline Urinproduktion & normal oder vermindert & vermindert & minimal \\
\hline Hautfalten & verstreichen sofort & verstreichen $(<2 \mathrm{~s})$ & verstreichen ( > 2s) \\
\hline Herzfrequenz & normal & normal bis erhöht & Tachykardie \\
\hline Atmung & normal & normal bis vertieft & tiefe Azidoseatmung \\
\hline Augen & normal & eingesunken & tief eingesunken \\
\hline Tränen & vorhanden & vermindert & fehlend \\
\hline Fontanelle & normal & leicht eingesunken & eingesunken \\
\hline
\end{tabular}


Bei Bedarf kann man nach beginnender Urinausscheidung und Elektrolytbestimmung auch eine Vollelektrolytlösung (Ringer) verwenden. Kinder unter 1 Jahr benötigen wegen ihrer geringen Energiereserven zudem eine Glukosezufuhr. Den Basisbedarf können Sie anhand der 4-2-1erRegel berechnen. Verluste sollen ebenfalls mit (glukosefreier) NaCl- oder Vollelektrolytlösung ersetzt werden ( $\bullet$ Tab. 3 ).

- Die Rehydrierung erfolgt bei Schock mit initial 20-60 ml/kg KG NaCl 0,9\% als Bolus intravenös.

- Bei schwerer Dehydration erfolgt der Ausgleich mit $\mathrm{NaCl}$ 0,9\% über $12 \mathrm{~h}$.

- Bei leichter bis mittelschwerer Dehydratation sollte der Flüssigkeitsverlust innerhalb von $6 \mathrm{~h}$ ersetzt werden.

Cave Die Regel muss bei jedem Patienten individuell überprüft werden. Insbesondere bei Patienten mit bekanntem Vitium cordis muss der Ausgleich des Defizits deutlich langsamer erfolgen.

\section{Hypo- und Hypernatriämie}

\section{$\nabla$}

Hyponatriämie Bei sonst gesunden Patienten kommt es in der Regel durch starken gastrointestinalen Verlust oder durch die Zufuhr hypotoner Lösungen zur Hyponatriämie. Wenn die Hyponatriämie langsam entstanden ist, muss ein langsames Anheben des Serum-Natriums um nicht mehr als 1-2 mmol/l pro Stunde erfolgen. Anderenfalls droht eine pontine Myelinolyse!

Normalerweise haben Kinder Natriumspiegel von etwa 133-146 mmol/l.

- Bei Serum-Natrium < $120 \mathrm{mmol} / \mathrm{l}$ verlegen Sie das Kind direkt auf die Intensivstation.

- Bei 120-130 mmol/l rehydriert man wie oben beschrieben, allerdings über $6 \mathrm{~h}$. Kontrollieren Sie das Natrium nach $1 \mathrm{~h}$.

- Bei zu raschem Anstieg reduziert man die Natriumzufuhr durch Mischen von $\mathrm{NaCl}$ 0,9\% mit Glukose 5\% - zunächst im Verhältnis 2/3 $\mathrm{NaCl}$ mit 1/3 Glukose (Natrium-Konzentration: $101 \mathrm{mmol} / \mathrm{l})$. Kontrollieren Sie nach $1 \mathrm{~h}$ und richten Sie ggf. die Infusion neu.

Ziel bei Hyponatriämie ist das Anheben des Serum-Natriums um 1-2 mmol// pro Stunde.
Hypernatriämie Die Hypernatriämie ist meist eine Folge von intestinalem Wasserverlust. Das folgende gilt daher nicht für Patienten mit Diabetes insipidus.

- Cave: Ein rascher Abfall des Serum-Natriums kann zu einem Hirnödem führen. Daher darf der Natriumspiegel nicht um mehr als $0,5 \mathrm{mmol} / 1$ pro Stunde sinken.

Initial sind engmaschige Kontrollen des SerumNatriums notwendig.

- Kinder miteinemSerum-Natrium > $160 \mathrm{mmol} / \mathrm{l}$ werden direkt auf die Intensivstation verlegt.

- Bei Werten von 150-160 mmol/l rehydrieren Sie wie oben beschrieben, allerdings über 8-12 h. Kontrolle des Serum-Natriums nach $1 \mathrm{~h}$.

- Bei zu raschem Abfall erhöht man die Natriumzufuhr durch Mischen von $\mathrm{NaCl} 0,9 \%$ mit $\mathrm{NaCl}$ $10 \%$ (über den Perfusor). $\mathrm{NaCl} 10 \%$ enthält $1,7 \mathrm{mmol} / \mathrm{l}$ Natrium. Erneut Kontrolle nach $1 \mathrm{~h}$, ggf. NaCl-Zufuhr anheben.

Ziel bei Hypernatriämie ist das Absenken des Serum-Natriums um $0,5 \mathrm{mmol} / \mathrm{l}$ pro Stunde. Bei zu langsamem Abfall des Serum-Natriums wird $\mathrm{NaCl} 10 \%$ reduziert.

\section{Bewusstseinstrübungen}

Ursachen Bei Kindern gibt es eine Menge von Differenzialdiagnosen, die mit einer Vigilanzstörung einhergehen. Als Ursache sind Befunde wie

- Fieber (Fieberkrampf, Sepsis, Meningitis),

- Kreislaufzentralisation (Schock) und

- Trauma möglich.

Notfälle mit Bewusstseinsstörungen als alleinigem Symptom sind weitaus seltener. Hierzu gehören

- Hyperglykämie (oft als Zeichen einer Erstmanifestation eines Diabetes mellitus) und

- Hypoglykämie im Rahmen einer Insulintherapie, durch Ernährungsfehler bei Diabetes mellitus oder selten auch bei Stoffwechseldefekten. Besteht Verdacht auf Hypoglykämie, ist aber kein Blutzuckertest möglich, soll bei einer Bewusstseinsstörung $1 \mathrm{ml} / \mathrm{kg} \mathrm{KG} \mathrm{20 \%} \mathrm{Glukoselösung} \mathrm{ge-}$ geben werden.

Berechnung des Basisvolumens zur Rehydrierung (4-2-1er-Regel)

Tab. 3 Mod. nach [3].

$4 \mathrm{ml} / \mathrm{kg} / \mathrm{h}$ für die ersten $10 \mathrm{~kg}$ KG plus

$2 \mathrm{ml} / \mathrm{kg} / \mathrm{h}$ für die zweiten $10 \mathrm{~kg}$ KG $(10-20 \mathrm{~kg})$ plus

$1 \mathrm{ml} / \mathrm{kg} / \mathrm{h}$ für jedes weitere $\mathrm{kg}$ über $20 \mathrm{~kg}$ KG

Beispiel: Ein $25 \mathrm{~kg}$ schweres Kind sollte $40+20+5=65 \mathrm{ml} / \mathrm{h} \mathrm{NaCl} 0,9 \%$ oder Vollelektrolytlösung erhalten.

Volumenersatz (z.B. als 20-ml-Boli): $\mathrm{NaCl}$ 0,9\% oder glukosefreie Vollelektrolytlösung, bei Kindern unter 1 Lebensjahr mit Glukose 1-2\%*

*Praxistipp 20 ml Glukose $40 \%$ zu 500 ml (Vollelektrolyt-)Lösung ergeben eine 1,6\% Glukoselösung. 


\section{Gehirnerschütterung}

In allen Altersklassen häufig Die Commotio cerebri ist ein häufiger Notfall - vom Säugling bis zum Schulkind. Für die Beurteilung ist die Glasgow Coma Scale sicher hilfreich. Mitberücksichtigen muss man immer

- das Alter des Kindes,

- woher es gerade kommt (Schule, Sport etc.),

- den Unfallhergang,

- Amnesie,

- Bewusstlosigkeit,

- äußere Verletzungen,

- insbesondere bei Säuglingen: Kontusionsmarken am Kopf und an den Fontanellen,

- Erbrechen und

- Schläfrigkeit.

Um eine mögliche Hirnblutung abzuklären, empfiehlt sich ein MRT bzw. CT des Schädels. Eine klinische Verlaufskontrolle in den ersten $48 \mathrm{~h}$ sollte gewährleistet sein.

\section{Fieberkrampf \\ $\nabla$}

Schwerwiegende Erkrankungen ausschließen Krampfanfälle gehören zu den häufigsten Notfällen im Kindesalter. Meist handelt es sich hierbei um Fieberkrämpfe. Auch wenn diese zum größten Teil harmlos sind, müssen Sie Differenzialdiagnosen wie Meningitis und Enzephalitis, SchädelHirn-Trauma und ausgeprägte Dehydratation ausschließen.

- Dazu gehört, dass alle Kinder < 1 Jahr wegen der überdurchschnittlich häufigen infektiösen Genese eine Lumbalpunktion erhalten.

- Eine Lumbalpunktion ist auch im 2. Lebensjahr sinnvoll, wenn kein klarer Fieberfokus erkennbar ist.

Der unkomplizierte einfache Fieberkrampf hat eine sehr gute Prognose.

- Beim komplizierten Fieberkrampf sollten auch bei älteren Kindern bis zum 2. Lebensjahr eine Lumbalpunktion und zusätzlich eine Bildgebung erfolgen. Das Epilepsierisiko ist in dieser Entität erhöht (๑ Tab. 4).

Alle Kinder unter 2 Jahren und alle Kinder jeglichen Alters mit erstmaligem kompliziertem Fieberkrampf müssen stationär eingewiesen werden.

Tab. 4 Aus [4]

\begin{tabular}{|l|l|l|}
\hline \multicolumn{2}{|l|}{ Unterschiede zwischen einfachen und komplizierten Fieberkrämpfen } \\
\hline Dauer & einfach & kompliziert \\
\hline Frequenz & einmalig & $>15$ min \\
\hline Ausdehnung & generalisiert & wiederholt innerhalb von 24h \\
\hline Häufigkeit & häufig $(>80 \%)$ & fokal \\
\hline Epilepsierisiko & gering $(2,5-3 \%)$ & weniger häufig (etwa 20\%) \\
\hline
\end{tabular}

Therapie des Fieberkrampfes Etwa 90\% aller Fieberkrämpfe enden von allein, ohne dass eine therapeutische Intervention notwendig ist.

- Dennoch wird die unverzügliche Unterbrechung des Anfalls durch Diazepam rektal (0,2$0,5 \mathrm{mg} / \mathrm{kg} \mathrm{KG}$ ) empfohlen.

- Bei Fortbestehen des Anfalls gibt man nach 5 min eine 2. Rektiole.

- Neben dieser Akuttherapie ist bei Fieber $>38,0^{\circ} \mathrm{C}$ eine antipyretische Therapie mit Paracetamol oder Ibuprofen indiziert.

Das weitere Vorgehen bei prolongiertem Fieberkrampf ist in $\diamond$ Abb. 1 dargestellt.

Infusion nur in schweren Fällen Persistiert der Krampfanfall nach 2-maliger Diazepam-Gabe über $10 \mathrm{~min}$, ist eine intravenöse Therapie mit Benzodiazepinen nötig.

- Da diese Medikamente atemdepressiv sind, müssen sie langsam und unter dem Schutz der Atemwege (Maskenbeatmung oder Intubation muss möglich sein) gegeben werden.

- Bei Säuglingen bis zu 1 Jahr ist Phenobarbital den Benzodiazepinen vorzuziehen.

Epileptische Patienten Kinder mit bekanntem Krampfleiden werden nach Notfallplan und Maßgaben des betreuenden pädiatrischen Neurologen behandelt und bei prolongierter Krampfdauer (Status epilepticus > 30 min) ebenfalls stationär eingewiesen. Bei diesen Kindern ist die Therapie oft komplex und individuell erstellt. Sie wird daher in dieser Notfall-Übersicht nicht näher erläutert.

\section{Diabetisches Ketoazidose-Koma}

Symptome Patienten mit einer diabetischen Ketoazidose fallen neben ihrer eingeschränkten Bewusstseinslage durch eine Kußmaulsche Atmung auf. Da es sich hierbei um ein lebensgefährliches Krankheitsbild mit dem Risiko für bleibende Hirnschäden handelt, ist eine rasche Diagnose und Therapie wichtig. Richtungsweisend sind - eine unphysiologisch langsame Atemfrequenz - und Azetongeruch.

- Häufig sind die Kinder auch dehydriert.

Therapie Ist das Zuckerkoma durch eine Blutzuckerbestimmung bestätigt,

- muss sofort eine Volumentherapie mit $\mathrm{NaCl}$ $0,9 \%$ (15-20 ml $/ \mathrm{kg} \mathrm{KG}$ ) über 30 min erfolgen.

- Anschließend stellt man die Flüssigkeitszufuhr entsprechend dem Grundbedarf und Defizit ein (s. o. unter "Exsikkose").

In Folge sollte der Blutzucker sinken und der Kaliumspiegel steigen. Ist das nicht der Fall, muss die Therapie des ketoazidotischen Komas stationär eskaliert werden:

- über Insulintherapie,

- Kaliumsubstitution und 
- unter engmaschigem neurologischem Monitoring (Glasgow Coma Scale).

Dies findet meist auf der Intensivstation statt, wo optimale Überwachungsbedingungen herrschen. Im präklinischen Notfalleinsatz sollte man eine Insulin- und Kaliumgabe möglichst vermeiden [2].

\section{Sepsis und septischer Schock}

Early-onset-Sepsis Im Jahre 2005 wurden die Kriterien einer Sepsis mit Organversagen für die Pädiatrie definiert [1]. Die Besonderheiten des Neugeborenen in den ersten Lebenstagen werden hier in den Vitalparametern (Atmung, Herzfrequenz und Blutdruck) abgebildet ( $\bullet$ Tab. 1). Gerade bei Neugeborenen ist die klinische Zuordnung zu einer Sepsis oft durch physiologische postpartale Änderungen und Differenzialdiagnosen erschwert, die bei der Diagnose und Therapie der Early-onset-Sepsis (innerhalb der ersten 3 Lebenstage) berücksichtigt werden müssen [5].

Mütterliche Risikofaktoren wie ein vorzeitiger Blasensprung, eine B-Streptokokken-Besiedelung oder eine Chorioamnionitis verpflichten uns, bei Anpassungsstörungen des Neugeborenen von einer Sepsis auszugehen.

Den oft unspezifischen klinischen Faktoren folgen dann noch diagnostische Laborparameter mit geringer Sensitivität [6]:

- CRP > $10 \mathrm{mg} / \mathrm{l}$

- IL-6 > 50/100 pg/ml (je nach Methode)

- IL-8 $>70 \mathrm{pg} / \mathrm{ml}$

- PCT $>1 \mathrm{ng} / \mathrm{ml}$ ( $>1$. Lebenswoche)

- $\mathrm{I} / \mathrm{T}>0,2(<1$. Lebenswoche $)$

- $\mathrm{NH}_{3}<100-150 \mu \mathrm{mol} / \mathrm{l}$ (Differenzialdiagnose)

Schnelles Handeln ist lebenswichtig Da die Early-onset-Sepsis bei reifen Neugeborenen sehr fulminant und bei Frühgeborenen noch ungünstiger verläuft, darf man bei Verdacht auf eine beginnende Sepsis keine Zeit verlieren.

- Beginnen Sie daher sofort eine antimikrobielle Behandlung mit einem Penicillin und einem Aminoglykosid/Cephalosporin.

Allerdings sollte bei klinischer Befundbesserung und bleibenden negativen Infektionsparametern innerhalb von 36-48 h die Antibiotikatherapie beendet werden [7].

Wenn nach klinischer Verschlechterung einer Infektion der Kreislauf mitbetroffen ist, spricht man von einem septischen Schock.

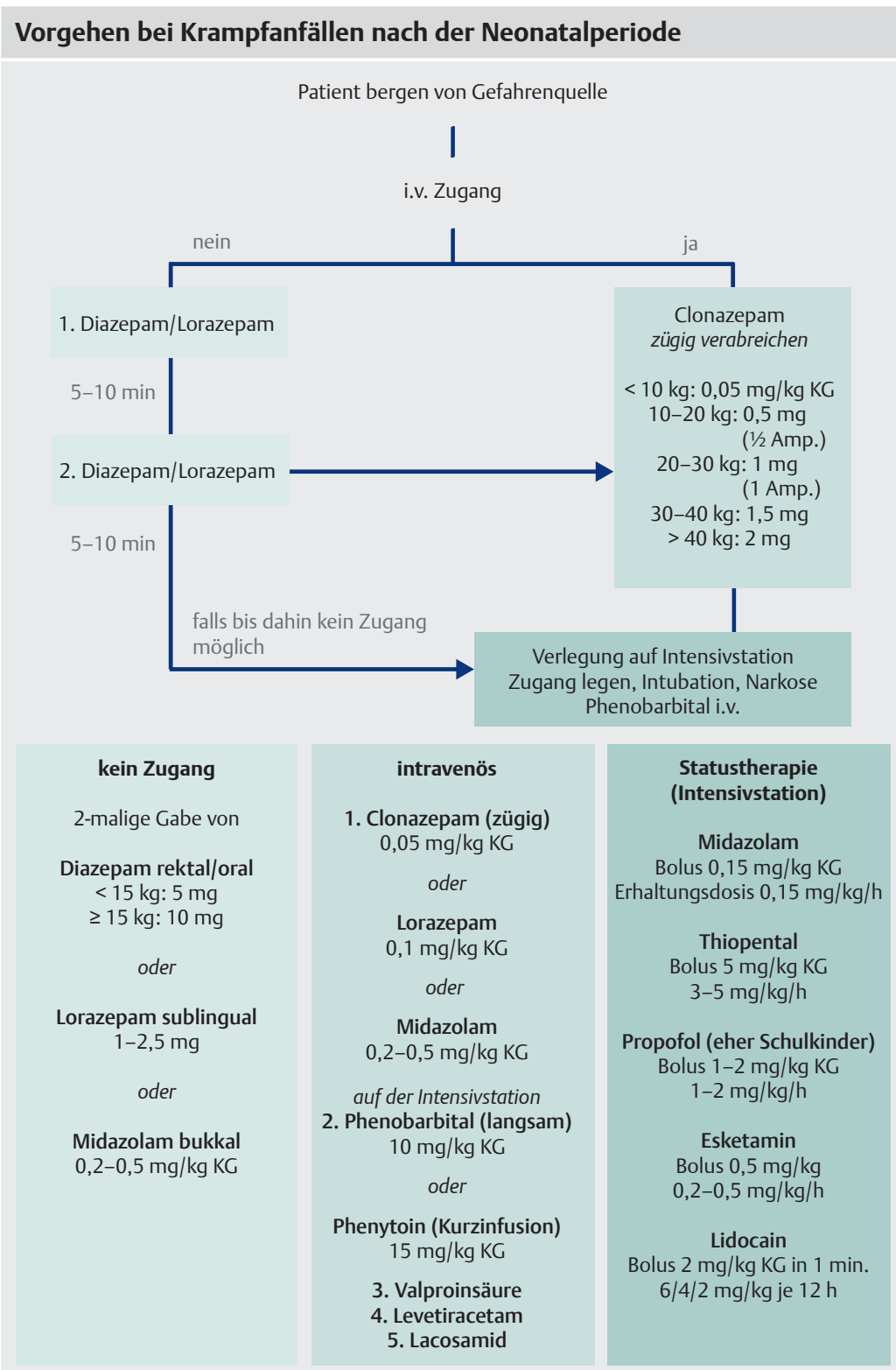

Merke: Die meisten Anfälle sistieren innerhalb von 5 min.

Je länger ein Anfall dauert, umso schwerer ist er zu durchbrechen. Behandlung eines Anfalls als Status epilepticus ab einer Dauer $>5$ min.

Seltene, aber wichtige Differenzialdiagnosen müssen ausgeschlossen werden: Schädel-Hirn-Trauma/Hirndruck, ausgeprägte Dehydratation, Intoxikationen, Hypoglykämie, Elektrolytentgleisung

Bei Fieber: Infektionen (Meningitis, Enzephalitis, Shuntinfektion, Sepsis) Erstmanifestation einer Epilepsie im Rahmen eines Infekts

Diagnostik bei erstem Krampfanfall oder Status epilepticus

obligatorisch: Blutgasanalyse, Blutzucker, $\mathrm{Na}, \mathrm{K}, \mathrm{Ca}, \mathrm{Mg}$, Infektparameter

bei erstem fokalem Ereignis: Bildgebung (MRT, CT, Sono)

fakultativ: Lumbalpunktion, Blut-/Urin-/Liquorkultur, Antikonvulsivaspiegel (Valproinsäure,

Phenobarbital, Phenytoin, Lamotrigin, Carbamazepin, Oxcarazepin), organische Säuren und Aminosäuren im Urin, Drogenscreening

Septischer Schock Den septischen Schock erkennt man an einer

- verlängerten Rekapillarisationszeit über $2 \mathrm{~s}$.

- Oft zeigen sich noch eine Tachykardie und/oder

- Hypotonie,

- Zyanose sowie

- Atemstörungen.
Abb. 1 Vorgehen bei Krampfanfall im Kindesalter (nach der Neonatalperiode). Es gelten die ABC-Richtlinien. Das Schema stammt aus der Klinik des Autors und dient dort als Leitfaden für die Ärzte. 
Abb. 2 Therapie des septischen Schocks bei Kindern (nach [9]). ECMO: extrakorporale Membranoxygenierung, FATD: femoralarterielle Thermodilution, Hb: Hämoglobin, MAP: mittlerer arterieller Druck, PAK: Pulmonalarterienkatheter, $\mathrm{ScVO}_{2}$ : zentralvenöse Sauerstoffsättigung,

ZVD: zentraler Venendruck, ZVK: zentraler Venenkatheter.

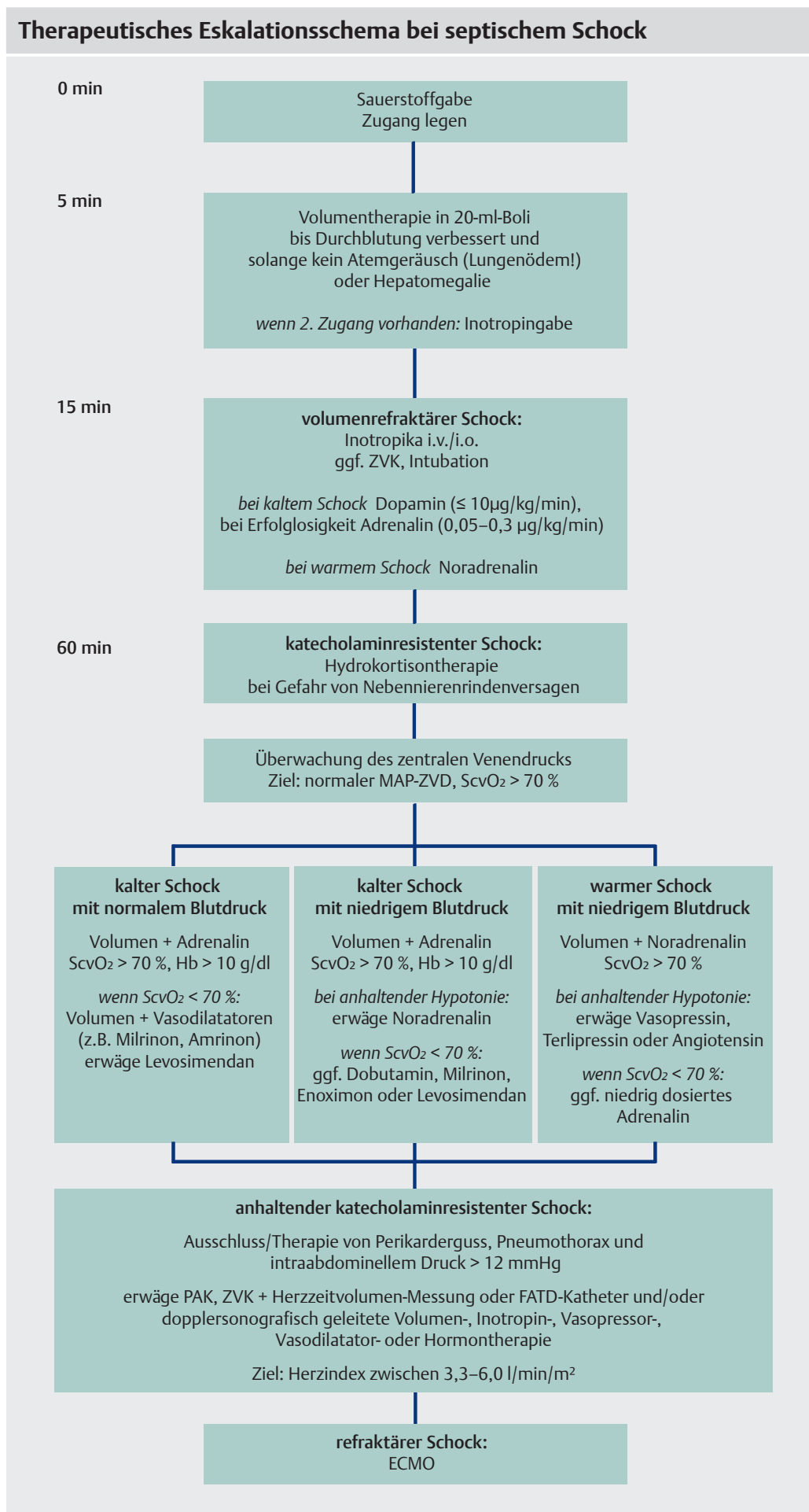

Hier ist primär eine sofortige Flüssigkeitsgabe bis zu $60 \mathrm{ml} / \mathrm{kg} \mathrm{NaCl}$ 0,9\% innerhalb $15 \mathrm{~min}$ indiziert. Der Erfolg lässt sich sehr gut am Blutdruck und noch besser an der Rekapillarisationszeit messen.

- Bei Versagen dieser Flüssigkeitstherapie muss ein Eskalationsschema entsprechend den Schockformen (kalt oder warm) über Dopamin, Dobutamin, Epinephrin, Typ-III-Phosphodiesterasehemmer (z. B. Milrinon) bis hin zur ECMO (extrakorporale Membranoxygenierung) folgen ( Abb. 2) [5, 8, 9].
Das schnelle Überwinden des Schocks mit ausreichend Flüssigkeit und ggf. nötiger Kathecholamin-Therapie innerhalb von 60 min verbessert die Überlebensrate um das 9-Fache [10].

Meningokokkensepsis Eine Sepsis kann durch Bakterien, Viren, Pilze und Protozoen verursacht werden. Besonders schlecht ist die Prognose bei einer Meningokokkensepsis: Sie verläuft in 30$90 \%$ der Fälle tödlich - oft binnen kürzester Zeit. Daher besteht extrem schneller Handlungsbedarf, und es ist unerlässlich, das klinische Bild einer Sepsis oder bakteriellen Meningitis zu erkennen:

- Unspezifische Zeichen wie Fieber, Erbrechen, Kopfschmerzen, Nackensteifigkeit, Apathie und in seltenen Fällen Krampfanfälle bei älteren Kindern.

- Trinkfaulheit, Hyperexzitabilität und vorgewölbte Fontanelle bei Neugeborenen und Säuglingen können dem foudroyanten Verlauf vorausgehen.

- Kommen zu diesen klinischen Zeichen noch Petechien in der Haut hinzu (nicht wegdrückbare Hauterscheinungen), muss sofort an eine Meningokokken-Sepsis gedacht werden.

Bei fortschreitendem Verlauf sollten Sie wie beim septischen Schock ( $\bullet$ Abb. 2) vorgehen.

Adjunktive Therapie bei Sepsis Zusätzlich zur kausalen und supportiven Therapie durch Antibiotika und Volumensubstitution verbessert eine adjuvante Therapie die Prognose der Patienten.

- Bei Frühgeborenen gibt es einen Überlebensvorteil unter Pentoxifyllin-Gabe (PTX, Dosis: 5 $\mathrm{mg} / \mathrm{kg} / \mathrm{h}$ für $6 \mathrm{~h}$ über 6 Tage) [11].

- Bei Meningokokkensepsis scheint nicht aktiviertes Protein C ohne schwerwiegende Nebenwirkungen zu Befundverbesserungen zu führen: In einer Dosis von $60-80 \mathrm{mg} / \mathrm{kg} 1$ - bis 2-mal täglich (je nach Plasma-Protein-C-Spiegel) zeigte sich an einer kleinen Fallguppe $<100$ Patienten ein positiver Effekt auf den Verlauf einer Purpura fulminans.

Aktiviertes Protein $\mathrm{C}$ wird dagegen bei Kindern und Neugeborenen nicht empfohlen [12]. Auch die Gabe von polyklonalen intravenösen Immunglobulinen (IVIG) ist nicht vorteilhaft [13]. Überzeugende Daten fehlen ebenso für den Einsatz des Granulozyten-Kolonie-stimulierenden Faktors G-CSF, für Steroide oder die optimale Einstellung des Blutzuckers.

\section{Ähnliche Krankheitsbilder \\ $\nabla$}

Pulmonale Hypertonie Oxygenierungsstörungen können neben einer Infektion auch durch einen physiologisch erhöhten Pulmonalarteriendruck als Zeichen einer persistierenden pulmonalen Hypertonie des Neugeborenen (PPHN) mit Rechtsherzversagen enstehen. 
Die Therapie erfolgt über:

- NO-Inhalation (20 ppm) über CPAP oder Intubation zur Vasodilatation

- Ausgleich einer Azidose mittels Bikarbonat

- Vermeidung höherer Dosen von Dopamin $>8 \mu \mathrm{g} / \mathrm{kg} / \mathrm{min}$, da sich sonst der periphere Gefäßwiderstand zusätzlich erhöht

- ECMO

Hyperammonämie Eine Hyperammonämie bei angeborenen Harnstoffzyklusdefekten geht mit einem Sepsis-ähnlichen Krankheitsbild einher. Bereits wenige Stunden nach der Geburt treten die typischen Zeichen auf:

- muskuläre Hypotonie,

- Erbrechen und

- Hyperventilation.

Als Folge der Stoffwechselstörung drohen irreversible Hirnschäden und der Tod. Daher müssen Sie bei kranken Neugeborenen mit unauffälligen Infektwerten oder unauffälliger Infektanamnese das Ammoniak $\left(\mathrm{NH}_{3}\right)$ bestimmen.

- Die Normwerte für $\mathrm{NH}_{3}$ liegen in der 1. Lebenswoche $<150 \mu \mathrm{mol} / \mathrm{l}$. Danach pendeln sie sich bei $25-50 \mu \mathrm{mol} / 1$ ein.

- Bei höheren Werten ist eine medikamentöse Stickstoffentgiftung (z.B. mit Natriumbenzoat) nötig.

- Eine Akut-Dialyse ist indiziert, wenn

$\triangleright$ der $\mathrm{NH}_{3}$-Spiegel $>400 \mu \mathrm{mol} / \mathrm{l}$ liegt oder sich das Kind im Koma befindet und

$\triangleright$ der $\mathrm{NH}_{3}$-Spiegel unter medikamentöser Therapie nicht innerhalb von $6-8 \mathrm{~h}$ sinkt.

Initialer $\mathrm{NH}_{3}[\mu \mathrm{mol} / \mathrm{l}] \times$ Komadauer $[\mathrm{d}]>4000$

hat eine schlechte Prognose!

Kardiogener Schock Vom septischen Schock des Neugeborenen muss der kardiogene Schock unterschieden werden. Er entsteht z.B. nach Spontanverschluss eines offenen Ductus Botalli bei Aortenisthmusstenose oder zyanotischen Vitien. Ein kardiogener Schock kann sich äußern in - Hepatomegalie,

- Zyanose,

- systolischem Herzgeräusch oder

- RR-Differenzen zwischen Arm und Bein.

Geben Sie bei V.a. Ductus-abhängigen kardiogenen Schock Prostaglandin E1 (50 ng/kg/min).

Fazit Die Auswahl dieser pädiatrischen Notfälle umfasst sowohl sehr häufige als auch seltene Krankheitsbilder. Wichtig sind Orientierungsschritte für die Therapiewahl und die Zeitabläufe. Der Hinweis auf Differenzialdiagnosen soll helfen, den richtigen Weg in der Behandlung nicht zu übersehen. Gerade in der Therapie des septischen Schocks verbessert das Einhalten des aktuell weltweit gültigen Schemas die Überlebenschancen wesentlich.

\section{Kernaussagen}

- Kindernotfälle unterscheiden sich von Notfällen im Erwachsenenalter. Während bei Erwachsenen kardiovaskuläre Ursachen im Vordergrund stehen, spielen bei Kindern v. a. respiratorische Probleme, Bewusstseinstrübungen und Dehydratation eine Rolle.

- Bei der Therapie von pädiatrischen Notfallpatienten gilt es, die Besonderheiten der kindlichen Anatomie, Physiologie und Psyche zu berücksichtigen.

- Bei respiratorischen Erkrankungen handelt es sich überwiegend um das KruppSyndrom und um Asthma.

- Bewusstseinstrübungen gehen zum größten Teil auf Fieberkrämpfe zurück. Sie verlaufen meist harmlos.

- Kinder können stark dehydriert sein, ohne dass man dies sofort bemerkt. Der Flüssigkeitsverlust kann das 3-Fache des zirkulierenden Blutvolumens betragen!

- Eine Sepsis kann v. a. bei kleinen Kindern (Neugeborenen) foudroyant verlaufen. Um Amputationen durch das Auftreten einer Purpura fulminans, einen septischen Schock oder den Tod des Kindes zu verhindern, ist schnelles Eingreifen notwendig.

- Therapeutische Mittel der Wahl sind

$\triangleright$ bei inspiratorischen Problemen: Adrenalin

$\triangleright$ bei exspiratorischen Problemen: $\beta 2$-Mimetika

$\triangleright$ bei unausgeglichenem Wasser- und Elektrolythaushalt: Volumentherapie mit $\mathrm{NaCl} 0,9 \%$ oder Vollelektrolytlösung

$\triangleright$ bei Fieberkrämpfen: Benzodiazepine

$\triangleright$ bei Sepsis: Antibiotika, Volumensubstitution und Inotropika; bei Frühgeborenen auch Pentoxifyllin

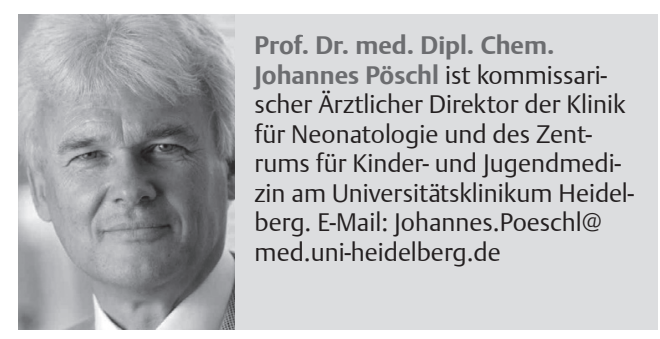

Interessenkonflikt Der Autor erklärt, dass keine Interessenkonflikte vorliegen.

Beitrag online zu finden unter http://dx.doi. org/10.1055/s-0031-1281450

\section{Literatur online}

Das vollständige Literaturverzeichnis zu diesem Beitrag finden Sie im Internet:

Abonnenten und Nichtabonnenten können unter „www.thieme-connect.de/ejournals“ die Seite der Lege artis aufrufen und beim jeweiligen Artikel auf „Ergänzendes Material“ klicken - hier ist die Literatur für alle frei zugänglich.

Abonnenten können alternativ über ihren persönlichen Zugang an das Literaturverzeichnis gelangen. Wie das funktioniert, lesen Sie unter: http://www. thieme-connect.de/ejournals/help\#SoRegistrieren 